\title{
A Hypothesis-Driven Association Study of 28 Nuclear-Encoded Mitochondrial Genes with Antipsychotic-Induced Weight Gain in Schizophrenia
}

\author{
Vanessa F Gonçalves'1,2, Clement C Zai' ${ }^{1,2}$, Arun K Tiwari², Eva J Brandl',2, Andriy Derkach ${ }^{3}$, \\ Herbert Y Meltzer ${ }^{4}$, Jeffrey A Lieberman ${ }^{5}$, Daniel J Müller, ${ }^{*, 1,2}$, Lei Sun ${ }^{*, 3,6}$ and James L Kennedy*, , 2 \\ 'Department of Psychiatry, University of Toronto, Toronto, ON, Canada; ${ }^{2}$ Neuroscience Section, Centre for Addiction and Mental Health, Toronto, \\ ON, Canada; ${ }^{3}$ Department of Statistical Sciences, University of Toronto, Toronto, ON, Canada; ${ }^{4}$ Department of Psychiatry and Behavioral \\ Sciences, Northwestern University, Chicago, IL, USA; ${ }^{5}$ Department of Psychiatry, College of Physicians and Surgeons, Columbia University and the \\ New York State Psychiatric Institute, New York City, NY, USA; ${ }^{6}$ Biostatistics Division, Dalla Lana School of Public Health, University of Toronto, \\ Toronto, ON, Canada
}

\begin{abstract}
Mitochondria are the main source of energy for neurons and have a role in many vital neuronal functions. Mitochondrial dysfunction has been described in schizophrenia, and antipsychotics such as clozapine and olanzapine have been associated with differences in gene expression in mitochondria. We investigated the hypothesis that nuclear-encoded mitochondrial genes, particularly those involved in oxidative phosphorylation or involved in oxidative stress, mitochondrial biogenesis, inflammation, and apoptosis, would be associated with antipsychotic-induced weight gain (AIWG). In total, we selected 28 genes and analyzed 60 SNPs (50 are functional), in 283 schizophrenia subjects, treated with atypical medications for up to 14 weeks. Association between AIWG (as measured by the \% of weight gain from baseline) and SNP genotypes were tested using linear regression with treatment duration, baseline body weight, and medication type as covariates. We observed a significant association between rs6435326 in the NDUFS I gene and AIWG in the subset of European patients $\left(N=150, P_{\text {corrected }}=0.02\right)$. The haplotype carrying the risk alleles of rs 6435326 and two other SNPs (rs I $0535 \mid 7$ and rs 180 I3।8) in NDUFS I was also nominally associated with percentage of weight gain (T-C-G vs A-T-A, $P=0.005$ ). In addition, stepwise linear regression was performed to select important variables predictive of the outcome, and a gene-gene interaction analysis was carried out. We observed a significant interaction between the TT risk genotype of rs6435326 in NDUFS I and AG genotype of rs3762883 in COXI $8\left(P_{\text {corrected }}=0.001\right)$. A permutation-based test of all 60 SNPs jointly showed significant association with weight gain $(P=0.02)$. Finally, our replication study of rs6435326, rs 1053517 and rs 1801318 in NDUFS I using samples from the Clinical Antipsychotic Trials of Intervention Effectiveness (CATIE) showed that $r$ I 801318 was significantly associated with AIWG $\left(N=200, P_{\text {corrected }}=0.04\right)$, and the three SNPs were collectively associated with AIWG $(P=0.04)$. In conclusion, our findings suggest an association between NDUFS I and AIWG in schizophrenia subjects. To the best of our knowledge, this is the first study to explore genetic variation in the mitochondrial genes in the context of AIWG.
\end{abstract}

Neuropsychopharmacology (2014) 39, 1347-1354; do::I0.1038/npp.2013.312; published online 26 February 2014

Keywords: mitochondria; weight gain; hypothesis driven; rs6435326; schizophrenia; clozapine

\section{INTRODUCTION}

Antipsychotics are the main treatment for ameliorating the symptoms of schizophrenia, although there is substantial

*Correspondence: Dr DJ Müller or Dr JL Kennedy, Department of Psychiatry, University of Toronto, 250 College Street, Clarke Site-, Rm 129, Toronto, ON M5T IR8, Canada, Tel: +4I6 979 4987, Fax: +4169794666, E-mail: daniel.mueller@camh.ca or jim.kennedy@ camh.ca or Dr L Sun Department of Statistical Sciences, University of Toronto, 6th Floor, Health Sciences Bldg, I 55 College Street, Toronto, ON M5T3M7, Canada. Tel: +416978 7519, Fax: +416978 8299, E-mail: sun@utstat.toronto.edu

Received I July 2013; revised 8 October 2013; accepted 22 October 2013; accepted article preview online 7 November 2013 inter-individual variability regarding dose, response and side effects. Antipsychotic-induced weight gain (AIWG) is a common side-effect of many antipsychotics and is particularly pronounced with clozapine and olanzapine contributing to a main reason for non-compliance (Lieberman et al, 2005). Twin and family studies have suggested that the heritability of AIWG is between $60 \%$ and $80 \%$ (Gebhardt et al, 2010), indicating that genetic factors have a significant role in its development. Indeed, several genetic and biological systems appear to influence AIWG (reviewed by Muller and Kennedy (2006) and Lett et al (2012)). However, the mitochondrial system has been underexplored despite its importance for body energy homeostasis and neuronal functions. Mitochondria are the main source of energy, and 
neurons, with their high level of differentiation and elevated metabolic rate, are very dependent on mitochondrial oxidative phosphorylation (OXPHOS) (Kann and Kovacs, 2007). This process of energy production occurs in the mitochondrial inner membrane through the respiratory chain, which contains five protein complexes. Besides ATP, reactive oxygen species (ROS) are also products of OXPHOS and are involved in intracellular signaling cascades, synaptic transmission and communication between neurons and glia (Kann and Kovacs, 2007).

Antipsychotic medications have been associated with differences in gene expression in mitochondria, although the molecular mechanisms (including a possible genetic predisposition) by which each drug alters mitochondrial function are poorly understood. Studies have suggested that antipsychotics such as haloperidol (first-generation antipsychotic FGA) may inhibit complex I activity of OXPHOS (Balijepalli et al, 2001). SGAs, such as clozapine and risperidone, also inhibit complex I activity but at a lower level than haloperidol (Balijepalli et al, 2001). Studies involving clozapine suggest that the mechanism by which the drug alters mitochondrial activity appears to involve oxidation of proteins and induction of oxidative stress in both human neuroblastoma cells and lymphoblastoid cell lines from schizophrenia subjects (Walss-Bass et al, 2008); (Baig et al, 2010). These studies suggest that oxidative stress may be one of the mechanisms by which clozapine increases risk for metabolic syndrome and diabetes (Baig et al, 2010). Olanzapine is not known to induce oxidative stress (Reinke et al, 2004) but has been associated with decreased fatty acid oxidation, which may predispose subjects to weight gain (Graham et al, 2005). Olanzapine has also been associated with increased levels of citrate synthase in pre-frontal cortex, hippocampus, and striatum in rats (Agostinho et al, 2011). This enzyme is critical for the Krebs cycle, as it catalyzes the first reaction of the pathway, condensing acyl groups from acetyl-CoA with oxalacetate to yield citrate. Finally, Choi et al (2009) compared the gene expression profiles of postmortem liver tissue between FGA and SGA groups of schizophrenia patients. The authors reported that 14 mitochondrial genes were differentially expressed between the two groups with 11 genes downregulated and 3 upregulated.

Given the above, we hypothesized that polymorphisms in genes involved in mitochondrial function may predispose individuals for weight gain after exposure to SGAs. Specifically, we selected 12 nuclear-encoded mitochondrial genes according to their involvement with OXPHOS. Furthermore, we also included 16 genes involved in oxidative stress, mitochondrial biogenesis, inflammation, and apoptosis that we propose may also be part of the mechanism by which SGAs lead to weight gain (Prabakaran et al, 2004; Konradi et al, 2004; Sun et al, 2006; Ben-Shachar and Karry, 2008; Gigante et al, 2011; Da Pozzo et al, 2012; Scola et al, 2013). Compared with the traditional genomewide association study (GWAS) design that requires very large sample sizes to achieve sufficient power, this hypothesis-driven (HD) approach allowed us to perform an association study with increased statistical power for prioritized genes (Sun et al, 2012). Nevertheless, power for any variants at the genome-wide level is limited by the small sample size even with the prioritization. Therefore, instead of using the original GWAS-HD approach proposed by Sun et al (2012), we used HD as a general principle focusing on the set of HD-selected 28 genes only.

\section{MATERIALS AND METHODS}

\section{Subjects}

Patients 18-60 years old with schizophrenia or schizoaffective disorder were diagnosed according to DSM-III-R or DSM-IV criteria and recruited from four different investigators (total sample $N=283$ ): DJM-1 (Berlin, Germany, $N=87$ ); HYM (Cleveland, USA, $N=61$ ); JAL (New York and North Caroline, USA, $N=74$ ); DJM-2 (Toronto, Canada, $N=61)$. A complete description of the study was provided to the participants and written informed consent was obtained in line with each institution's ethics review board guidelines. In the case of DJM-1, patients were prescribed SGAs and assessed for up to 6 weeks. For samples HYM and JAL, patients did not have any prior exposure to SGAs and were treated for 6 weeks, or up to 14 weeks, respectively. For sample DJM-2, patients were prescribed antipsychotic medication and followed up for a minimum of 6 weeks. Demographic and clinical characteristics of the sample stratified by the four sub-samples are provided in Table 1.

\section{Genotyping}

Single-nucleotide polymorphism selection. Genomic DNA was extracted from blood samples using the highsalt method (Lahiri and Nurnberger, 1991). For this study, we genotyped only individuals with European ancestry $(N=183)$. For the 28 nuclear-encoded mitochondrial genes selected based on our hypothesis, gene boundaries included $5 \mathrm{~kb}$ in $5^{\prime}$ and $2 \mathrm{~kb}$ in $3^{\prime} \mathrm{UTR}$ regions. For each gene, tagging SNPs were selected using HapMap database, phase + phase3, release \#28, CEU population, Build36 (www. hapmap.org) and Tagger in Haploview (Barrett et al, 2005). The threshold for the minor allele frequency (MAF) was set at 0.05 . In total, 64 SNPs were selected according to MAF $>0.05$ and for tag SNPs, linkage disequilibrium (LD, $\left.r^{2} \geqslant 0.8\right)$.

Most of the SNPs selected (50 out of 60 SNPs) in this study were classified as functional according to Haploreg (Ward and Kellis, 2012) and SNPinfo (Xu and Taylor, 2009). Each of the 28 genes has at least one functional SNP. For 8 out of these 28 genes, we also included non-functional SNPs due to two main reasons: 1) The gene had only one functional SNP tagged by our approach and, thus, we decided to include non-functional SNPs for better coverage of these genes. 2) The gene has shown differential gene expression in schizophrenia patients and we decided to take all tag SNPs for them (including both functional and nonfunctional). Details about the SNP selected are provided in Supplementary Table 1.

Genotyping and quality control (QC). Genotyping was performed on the TaqMan Openarray AccuFill System (Life Technologies, NY, USA) using 64 SNP per array. Briefly, $2.5 \mu \mathrm{l}$ of DNA $(50 \mathrm{ng} / \mu \mathrm{l})$ was mixed with $2.5 \mu \mathrm{l}$ of the TaqMan OpenArray Master Mix $(40 \times)$ onto the OpenArrays 384-well sample plate. The OpenArray plate was 
Table I Demographic and Clinical Characteristics of the Samples of the Study

\begin{tabular}{|c|c|c|c|c|c|c|}
\hline & DJM-I & HYM & JAL & DJM-2 & Total sample & $P$-value \\
\hline Number of individuals & 87 & 61 & 74 & 61 & 283 & \\
\hline Weight gain (\%) & $4.0 \pm 4.6$ & $4.8 \pm 6.3$ & $5.9 \pm 7.9$ & $4.8 \pm 10.6$ & $4.84 \pm 7.22$ & $0.50^{\mathrm{a}}$ \\
\hline Baseline weight (kg) & $81.1 \pm 15.8$ & $76.5 \pm 13.9$ & $82.4 \pm 17.5$ & $82.8 \pm 18.8$ & $80.5 \pm 16.72$ & $0.28^{\mathrm{a}}$ \\
\hline Age (years) & $35.2 \pm 12.5$ & $33.4 \pm 8.9$ & $39.31 \pm 8.1$ & $36.9 \pm 12.2$ & $36.1 \pm 10.7$ & $<0.008$ \\
\hline Study duration (weeks) & $5.1 \pm 1.59$ & 6.00 & $10.4 \pm 4.2$ & $3.7 \pm 1.7$ & $6.4 \pm 3.45$ & $<0.001^{\mathrm{a}}$ \\
\hline Male & 55 (63.2\%) & 42 (68.9\%) & $56(75.7 \%)$ & 42 (68.9\%) & 195 (68.9\%) & \\
\hline Female & $32(36.8 \%)$ & $19(31.1 \%)$ & $18(24.3 \%)$ & $19(31.1 \%)$ & $88(31.1 \%)$ & \\
\hline \multicolumn{7}{|l|}{ Race } \\
\hline European & 85 (98\%) & 42 (68.9\%) & $26(35.1 \%)$ & $30(49.2 \%)$ & $183(64.7 \%)$ & $<0.001$ \\
\hline Olanzapine & $15(\mid 7.2 \%)$ & & $22(29.7 \%)$ & $8(13.1 \%)$ & 45 (I5.9\%) & \\
\hline Others & $62(71.3 \%)$ & & $25(33.8 \%)$ & $12(19.7 \%)$ & 99 (35.0\%) & \\
\hline Individuals genotyped ${ }^{\mathrm{b}}$ & 80 & 38 & 17 & 29 & $164(89.6 \%)$ & \\
\hline
\end{tabular}

${ }^{a}$ Kruskal-Wallis test.

${ }^{\mathrm{b}}$ Only Europeans were included in the study.

loaded into OpenArray case filled with immersion fluid, sealed with glue and, submitted to PCR. The cycling conditions were: initial denaturing at $93{ }^{\circ} \mathrm{C} 10 \mathrm{~min}$, following by 50 cycles at $95^{\circ} \mathrm{C}$ for $45 \mathrm{~s}, 94^{\circ} \mathrm{C}$ for $13 \mathrm{~s}$ and $53^{\circ} \mathrm{C}$ for $2 \mathrm{~min} 14 \mathrm{~s}$, and a final extension at $25^{\circ} \mathrm{C}$ for $2 \mathrm{~min}$. After PCR, the OpenArray plate was submitted for image at OpenArray Real-Time PCR Instrument (Biotrove).

Data quality control (QC) excluded four SNPs with poor clustering in allelic discrimination plots. Using PLINK (Purcell et al, 2007), none of the remaining SNPs had call rates $<95 \%$ or violated Hardy-Weinberg equilibrium $(P<0.01)$. Duplicate samples $(15 \%)$ were used to check genotyping accuracy, and $100 \%$ concordance was observed. QC for individuals excluded 19 samples with $>5 \%$ missing genotype rate or high/lower heterozygosity ( $\pm 3 \mathrm{SD}$ from the sample mean). In total, 60 SNPs and 164 individuals passed the QC criteria and were kept for further analyses. The exact number of individuals used in each of the statistical analyses varied depending on the missing data rate of the covariates involved in that analysis.

\section{Statistical Analyses}

For detecting heterogeneity between study samples, categorical variables were compared using Pearson $\chi^{2}$ test, and continuous variables were analyzed using Student $t$-test or analysis of variance (SPSS 15.0, SPSS, Chicago, IL, USA). Linear regression was used to test the association, where percentage of weight gain from baseline was the dependent variable, and genotypes, baseline weight, study duration and medication type were predictors. The genotypes were coded additively as 0,1 and 2, representing the number of copies of the minor allele. The 'medication type' variable included clozapine and olanzapine as highest risk for weight gain (1) and the other drugs as low risk (0, the reference category) (Tiwari et al, 2013). The outliers for weight gain were winsorized at the $99 \%$ level for better normal approximation of the data (Supplementary Figure 1). UNPHASED version 3.1.5 (Dudbridge, 2003) was used for haplotype comparisons, and all haplotypes with frequency $\geqslant 5 \%$ were included in the analyses. Haploview was used to display pair-wise LD between SNPs. Corrections for multiple tests were performed using Single-Nucleotide Polymorphism Spectral Decomposition (SNPSpD) (Nyholt, 2004). Power calculation was performed using Quanto 1.2.4 (Gauderman and Morrison, 2006). Stepwise linear regression was carried out using SPSS to identify predictive variables that influence the outcome (percentage of weight gain from baseline). Genegene interaction between rs6435326 and SNPs identified from stepwise linear regression was performed using the $m b m d r$ software and significance was calculated using permutation test with 10000 simulated replicates (Calle et al, 2010).

Statistical evaluation of our biological hypothesis. Our hypothesis was that nuclear-encoded mitochondrial genes, particularly those involved in OXPHOS, oxidative stress, mitochondrial biogenesis, inflammation and apoptosis, are more likely to be associated with AIWG in schizophrenia subjects. To evaluate the statistical significance of this hypothesis (ie, does the association evidence of this set of prioritized genes, collectively, exceed what is expected under the null of no association), we performed a permutation-based test as in Sun et al (2012). Briefly, the weight gain (\%) phenotype and all covariates but the genotype were permuted jointly within the site and independently 1000 times (R; Team RC (2012)). For each permuted sample, corresponding association analysis was 
performed and a sum of the Wald association statistic (QUOTE distributed) of the $60 \mathrm{SNPs}$ was obtained. The empirical $P$-value was calculated as the proportion of the permutation samples whose sum of statistic was larger than that in the observed sample.

Replication of the findings. To replicate our findings (ie, rs6435326, rs1053517, and rs1801318 in NDUFS1), we used samples from the Clinical Antipsychotic Trials of Intervention Effectiveness (CATIE) study (Lieberman et al, 2005). This sample consisted of 741 chronic schizophrenia patients (18-65 years of age) diagnosed with schizophrenia according to Structured Clinical Interview for DSM-IV (SCID). For our replication study, we selected 200 individuals who satisfied the following criterion: subjects of European ancestry (based on population stratification analysis); subjects with $\mathrm{BMI}<40$ at baseline; subjects not previously treated with olanzapine or clozapine for more than 30 days before the CATIE study trial.

The three SNPs of interest (rs6435326, rs1053517, and rs1801318) were not genotyped in the CATIE GWAS sample. Thus, we imputed the genotypes using IMPUTE v2.2 (Howie et al, 2012) with the 1000 Genomes Project Phase 1 (March 2012) as the reference panel. While rs1053517 and rs1801318 had good imputation accuracy (IMPUTE INFO $>0.9$ ), rs6435326 had a call-rate $<95 \%$, and we used TaqMan SNP Genotyping assays (Applied Biosystems, Foster City, CA) to obtain reliable genotype data for this SNP.

The association analysis was then conducted using a similar method as in the discovery study. Briefly, the phenotype was defined as the percentage of change in BMI from baseline, genotype was defined as number of copies of the minor allele weighted by the genotype imputation posterior probabilities (ie, genotype 'dosage'), and covariates consisted of treatment duration (in days) and medication type (olanzapine as higher risk, and risperidone and quetiapine as lower risk). The multi-SNP joint analysis of all three SNPs was performed using the exact permutation method as described above.

\section{RESULTS}

Comparison among the four samples showed statistical differences in several demographic variables, however, we did not observe significant differences in the amount of weight gain across the samples, even after adjustment for duration (DJM-1 4.0 $\pm 4.6 \mathrm{SD}$, HYM $4.8 \pm 6.3 \mathrm{SD}$, JAL $5.9 \pm 7.9$ SD, DJM-2 4.8 \pm 10.6 SD, $P=0.20$ ). Thus, we decided to pool the samples for the analysis. In terms of power, assuming $\mathrm{MAF}=0.15$ and sample size of $N=150$ (only individuals with European ancestry and with complete covariates information), we had more than $80 \%$ power to detect a mean difference of $2.6 \%$ weight gain between carriers and non-carriers of the risk genotype in the additive model at the type 1 error rate of 0.05 .

We observed a significant association between weight gain $(\%)$ and $\operatorname{rs6435326}(N=150, \beta=-2.19, P=0.0003)$. TT homozygotes gained significantly less weight $(1.1 \pm 4.5 \%$ $\mathrm{SD} ; N=36)$ than heterozygotes $(3.1 \pm 4.5 \% \mathrm{SD} ; N=63)$ and AA homozygotes $(5.1 \pm 5.1 \% \mathrm{SD} ; N=51)$ (Figure 1). This SNP is located in an intronic region of the NDUSF1 gene

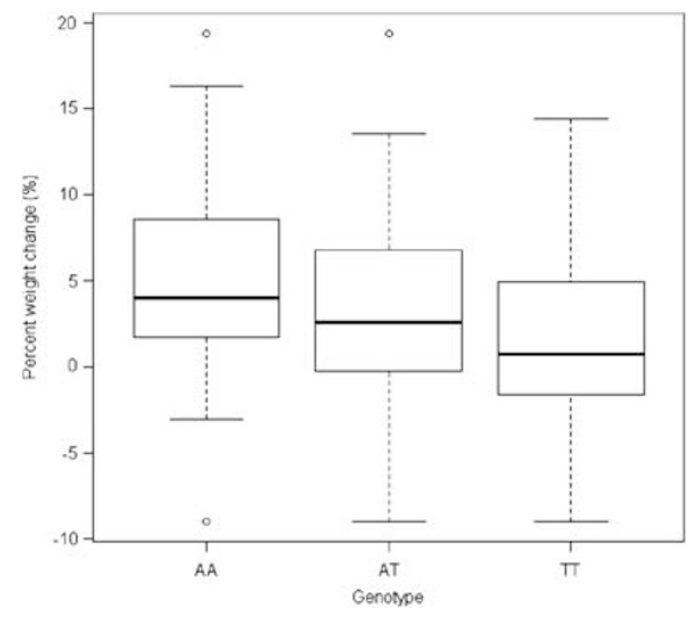

Figure I Box-plot graph showing the percentage of weight gain for genotype groups of the SNP rs6435326 in the NDUFSI gene. The boxes drawn are the median values for percentage of weight change, and the whiskers drawn correspond to minimum and maximum values observed. The outliers were defined as points more than 1.5 times of upper or lower quartiles.

(NADH dehydrogenase (ubiquinone) Fe-S protein 1, $75 \mathrm{KDa}$ ) which is part of the complex I of OXPHOS. To correct for multiple comparisons, we calculated the effective number of independent tests using SNPSpD (Nyholt test; Nyholt (2004)). The number of independent tests was 53 and association between rs6435326 and weight gain (\%) remained significant after correction $\left(P_{\text {corrected }}=0.02\right)$, consistent with the permutation-based adjusted $P$-value of 0.02 . To dissect the association evidence observed for rs6435326, sample-specific association analysis was performed, separately, for samples DJM-1, HYM, and DJM-2 (Supplementary Table 2). The size of sample JAL was too small for meaningful statistical analysis. The frequencies of minor allele $(\mathrm{T})$ were similar across all three samples and the protective effect of rs6435326 was observed in all three as well (Supplementary Table 2 and Supplementary Figure 2).

Two SNPs, rs1053517 and rs1801318, were moderately correlated with $\mathrm{rs} 6435326\left(r^{2}=0.5, D^{\prime}=0.95\right.$ and $r^{2}=0.3$, $D^{\prime}=1$, respectively) (Figure 2). Haplotype analyses revealed that the block carrying the risk alleles of rs6435326, rs 1053517 , and rs 1801318 was also significantly protective against weight gain (\%) (T-C-G vs $\mathrm{A}-\mathrm{T}-\mathrm{A}, P=0.005$ ). The stepwise linear regression analysis selected rs6435326 in NDUFS1 and rs3762883 in COX18. The gene-gene interaction analysis identified a significant interaction between rs6435326 and rs3762883 (10000 permutations, $P=0.001)$. TT homozygotes at rs6435326 and heterozygotes at rs3762883 were classified as low risk (least weight gain) $(\beta=-5.39, P=0.0006)$.

Given the small sample size and anticipated modest effect of individual genetic variants associated with this complex trait, it was not surprising that no other SNP, beyond rs6435326 in NDUSF1, was significantly associated with weight gain (\%). However, alternative analysis that evaluates the cumulative evidence of multiple SNPs can reveal association that might not be detectable at the individualSNP level. Indeed, our permutation-based test of all 60 SNPs 
a

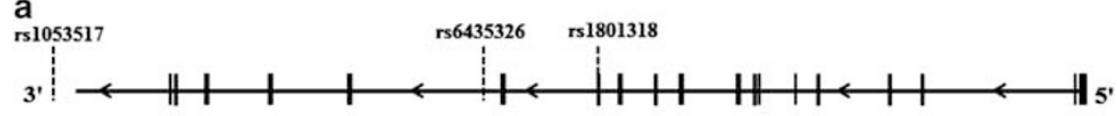

b

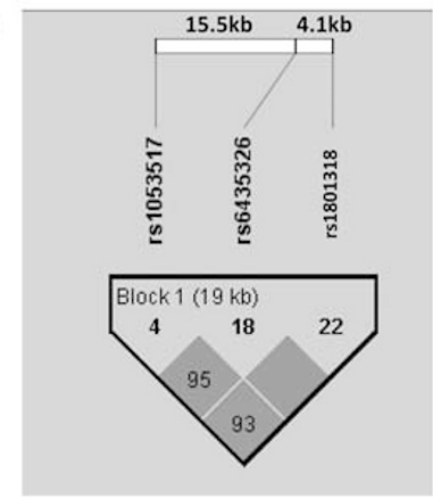

Figure 2 NDUFSI gene structure location of polymorphisms (a) and LD graph (b) for the SNPs genotyped. In panel a, the black boxes represent the exons and the width correspond to their sizes. In panel B, the values in the LD plot are D' values.

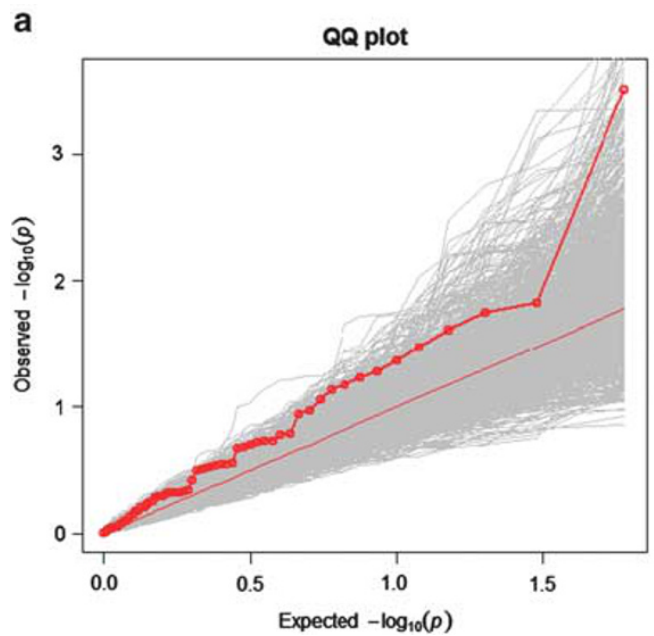

b $P=0.02 \quad$ Histogram for sum of $Z^{\wedge} 2$

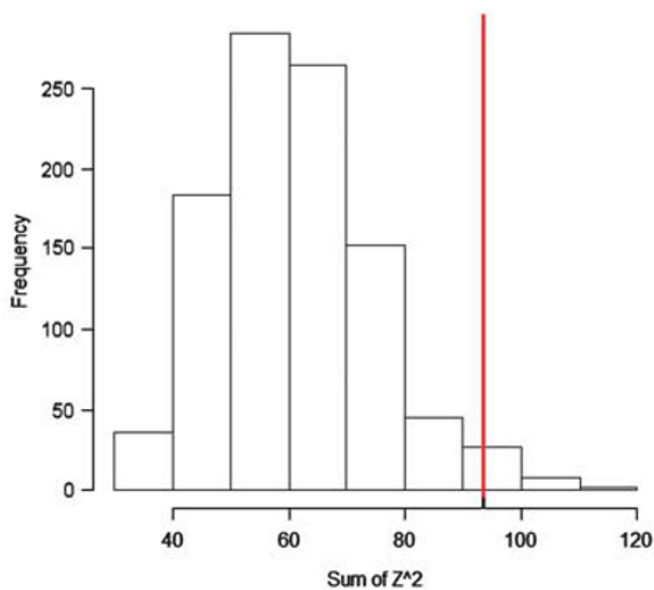

Figure 3 Mitochondria hypothesis testing. (a) QQ plot of the mitochondrial SNPs association evidence in the sample. Red curve correspond to the observed $P$-values. Gray curves correspond to the $P$-values obtained from the 1000 permutation replicates as described in the text. (b) Statistical significance of the mitochondria hypothesis. The statistical significance (permutation $P=0.02$ ) was calculated by comparing the observed sum association statistic (red vertical line) aggregated over all 60 SNPs with the sum statistics calculated from the 1000 permutation replicates (shown as the histogram).

Table 2 Replication of the Top Three SNPs

\begin{tabular}{|c|c|c|c|c|c|c|c|c|}
\hline \multirow[t]{2}{*}{ Marker } & \multirow[t]{2}{*}{ Gene } & \multirow[t]{2}{*}{ MAF } & \multirow[t]{2}{*}{ LD $^{\mathbf{a}}$} & \multicolumn{2}{|c|}{ Discovery sample $(n=164)$} & \multicolumn{3}{|c|}{ Replication sample $(n=200)$} \\
\hline & & & & $\beta$ & $\boldsymbol{P}$ & $\beta$ & $\mathbf{P}$ & INFO $^{b}$ \\
\hline rs 1053517 & NDUFSI & 0.43 & 0.8 & -1.45 & 0.018 & -1.98 & 0.04 & 0.94 \\
\hline$r s \mid 801318$ & NDUFSI & 0.30 & 0.4 & -1.47 & 0.033 & -2.29 & 0.02 & 0.94 \\
\hline
\end{tabular}

aMeasured by $r^{2}$ between the SNP and rs6435326.

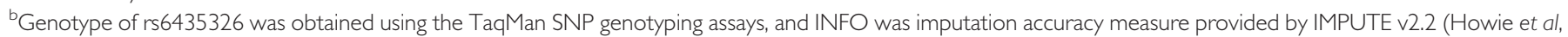
2012).

jointly showed that the 28 nuclear-encoded mitochondrial genes selected based on our hypothesis, collectively, were associated with weight gain $(P=0.02$, Figure 3$)$.
In the replication sample, the directions of effect for all top three SNPs in NDUFS1 (rs6435326, rs1053517, and rs1801318), were consistent with the discovery sample 


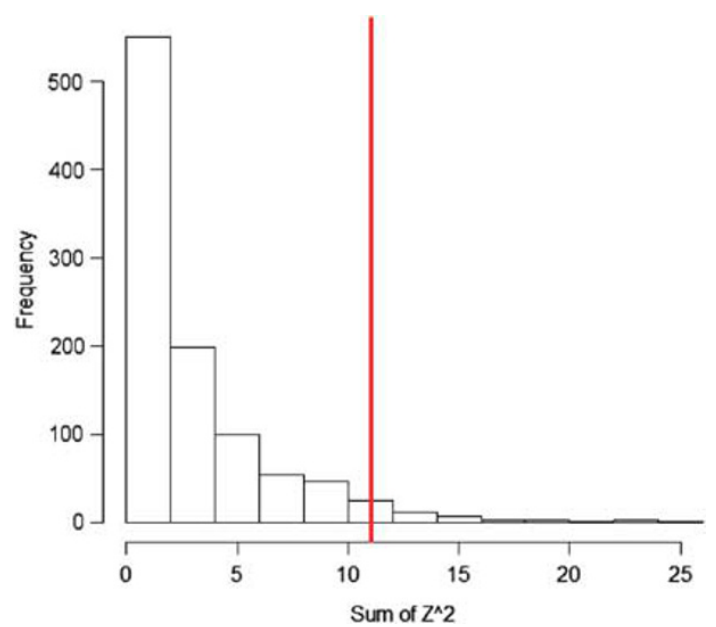

Figure 4 Replication of the association between NDUFSI and AIWG. The statistical significance $\left(P_{\text {permutation }}=0.04\right)$ was calculated by comparing the observed sum association statistic (red vertical line) aggregated over the three SNPs within NDUFS I with the sum statistics calculated from the 1000 phenotype permutation replicates (shown as the histogram).

(Table 2). Two of the three SNPs had replication $P$-values less than $0.05, \quad \operatorname{rs} 1053517 \quad(P=0.04)$ and $\operatorname{rs} 1801318$ $(P=0.02)$, but only one survived adjustment of multiple hypothesis testing $(<0.05 / 2=0.025$, where 2 is the number of independent tests among the 3 tests performed). However, the multi-SNP analysis of all three SNPs simultaneously in the CATIE replication sample provided convincing evidence that NDUFS1 is associated with AIWG $\left(P_{\text {permutation }}=0.04\right.$, Figure 4$)$.

\section{DISCUSSION}

In this study, we analyzed 60 SNPs in 28 nuclear genes involved in mitochondrial function, for association with AIWG, using the HD approach described by Sun et al (2012). Our HD analysis is technically different from what was originally proposed in Sun et al (2012), but the principle is the same. Here, instead of performing a genome-wide study, we decided to use the HD principle to select a small set of variants for a more focused study, providing sufficient power for these HD-selected variants given the small sample size.

Our hypothesis was that nuclear-encoded mitochondrial genes, particularly those from the OXPHOS pathway or those involved in oxidative stress, mitochondrial biogenesis, inflammation and apoptosis, are more likely to be associated with AIWG in schizophrenia subjects. To the best of our knowledge, this is the first study to explore genetic variation in the mitochondrial genes in the context of AIWG. In the single-SNP analysis, we observed significant association between rs6435326 and weight gain (\%), even after correction for multiple testing. The haplotype containing the $\mathrm{T}$ allele of rs6435326, C allele of rs1053517, and $\mathrm{G}$ allele of rs 1801318 was also significantly protective against weight gain. These SNPs are located in NDUFS1, which is part of the complex I of OXPHOS. NDUFS1 encodes the largest and one of the core subunits of this complex and the protein is located in the iron-sulfur fragment of the enzyme (Smeitink et al, 1998). NDUFS1 is part of the hydrophilic arm of the complex, which is responsible for the transfer of electrons (Finel, 1998; Scola et al, 2013). Mutations in this gene have been associated with isolated complex I deficiency (Hoefs et al, 2010); and it was proposed that dysfunction in the cellular oxidative metabolism leads to increased mitochondrial ROS (mROS) production (Iuso et al, 2006).

The effect of variants on mROS production may be of special importance, as it may influence the energy homeostasis in the hypothalamus. Recently, compelling evidence has shown the role of mROS for 'fuel sensing' in the melanocortin system in the hypothalamus (Leloup et al, 2006). Briefly, it was proposed that mROS is an important signaling pathway for stimulation of both the main types of neurons of the arcuate nucleus: 1) those that express anorectic pro-opiomelanocortin (POMC), and 2) orexigenic neurons that contain neuropeptide Y (NPY) and the agoutigene-related transcript (AgRP). In POMC neurons, leptin starts the process of depolarizing the neuronal membrane via opening of non-specific cation channels and activating the STAT3/JAK2 signaling pathway that leads to increased $P O M C$ gene expression. Also, during elevated blood glucose levels, glycolysis is active and OXPHOS produces mROS and ATP. It is proposed that the increase in mROS levels is sensitive to the closure of $\mathrm{K}_{\mathrm{ATP}}$ channels, a crucial step in keeping POMC neurons active. The cleavage of POMC triggers a chain of downstream events that will decrease food intake and increase energy expenditure in humans (reviewed by Horvath et al (2009) and Jordan et al (2010)). In NPY/AgRP neurons, ghrelin activates the AMPK signaling pathway, leading to inhibition of the acetyl-CoA carboxylase enzyme (ACC). As a consequence, the levels of malonyl-CoA decrease and activate the carnitine_palmitoyl_transferase I (CPT1) enzyme, which is responsible for transport of long-chain fatty acids into the mitochondrial matrix. Lower levels of glucose and high levels of fatty acid in the blood stream stimulate $\beta$-oxidation in mitochondria, increasing mROS production that leads to the expression of uncoupling protein 2 (UCP2). This protein acts by buffering mROS to keep gene expression active, allowing NPY to stimulate food intake and reduce energy expenditure. The $\mathrm{AgRP}$ acts as an antagonist of melanocortin 3 receptor and melanocortin 4 receptor blocking their anorectic effects (reviewed by Horvath et al (2009)).

Our current study also reports the significant interaction between the TT genotype of rs6435326 (NDUFS1) and AG genotype of rs3762883 (COX18). The variant, rs3762883, is predicted to be functional (DNAse hypersensitive, Haploreg v2) and COX18 is involved in the mitochondrial biogenesis and in the assembly of the complex IV of OXPHOS in the inner membrane (Gaisne and Bonnefoy, 2006). Complex IV is responsible for the final transfer of electrons to oxygen. Reduction in activity for both complex I and IV was identified in postmortem brain of schizophrenia subjects (Maurer et al, 2001). Thus, it is possible that variants in genes that form complex I and IV may predispose individuals for the side-effect of weight gain after SGA exposure.

In support to our hypothesis, we observed significant association evidence from a multi-SNP model that jointly analyzed all 60 variants in these 28 mitochondrial genes (permutation 
$P=0.02)$. The result suggests the involvement of multiple associated variants, each with small effect. A similar situation may exist regarding the polygenetic inheritance reported for schizophrenia (Gottesman and Shields, 1967).

Our study had greater than $80 \%$ power to detect the mean differences observed for rs6435326 at the type 1 error level of 0.05. Among the three top SNPs (rs6435326, rs1053517, and rs1801318 in NDUFS1), rs6435326 was not statistically significant $(P=0.18)$ in the CATIE replication sample. However, direction of effect is consistent with the discovery sample. Furthermore, the gene-based replication study (joint analysis of all three SNPs) replicated $(P=0.04)$ the finding that NDUFS1 is associated with AIWG.

In conclusion, our finding suggests the role of NDUFS1 on AIWG in schizophrenia subjects. Moreover, this study provides evidence implicating the mitochondria as a system involved in the regulation of energy homeostasis and body weight in schizophrenia subjects under SGA treatment.

\section{FUNDING AND DISCLOSURE}

VFG receives the CIHR Strategic Training for Advanced Genetic Epidemiology (STAGE) fellowship, University of Toronto. CCZ is supported by American Foundation for Suicide Prevention (2PDF-00065-1208-0609-1209), Eli Lilly, and Brain and Behavior Research Foundation (NARSAD). AKT is supported by Brain and Behavior Research Foundation (NARSAD). AD receives the Ontario Graduate Scholarship (OGS) and the CIHR Strategic Training for Advanced Genetic Epidemiology (STAGE) fellowship, University of Toronto. DJM is supported by Canadian Institutes of Health Research (CIHR Operating grant: Genetics of antipsychotics induced metabolic syndrome, MOP 89853). $\mathrm{He}$ was awarded with Brain and Behavior Research Foundation (NARSAD) Award, CIHR Michael Smith New Investigator Salary Prize for Research in Schizophrenia, Ontario Mental Health Foundation New Investigator Fellowship and an Early Researcher Award by the Ministry of Research and Innovation of Ontario. The research of LS is supported by the Natural Sciences and Engineering Research Council of Canada (NSERC) and CIHR. JLK received honoraria from Roche, Novartis, and Lilly, and he is a member of the scientific advisory board (unpaid) of AssureRx Health Inc. HYM has received grants, or payment for lectures, or is or was a consultant to: Sunovion Novartis, TEVA, Bioline Rx, BI, Envivo, Compainon Dx, Janssen Pharma, Lundbeck. HYM employment includes Suregene and Northwestern University, and he is expert testimony for Janssen. HYM is a shareholder of ACADIA, Glaxo Smith Kline and Suregene. HYM is a speaker or board member of Sunovion, TEVA, Bioline Rx, Janssen, BI, and is an advisor to Centerstone Research Institute. HYM received honoraria from Lundbeck, BI, Sunovion, TEVA, Bioline Rx, and Janssen Pharma. JAL serves on the Advisory Board of Intracellular Therapies. He does not receive direct financial compensation or salary support for participation in research, consulting, or advisory board activities. He receives grant support from Allon, Biomarin, Genentech, GlaxoSmithKline, Eli Lilly, Novartis, Psychogenics, Sepracor (Sunovion) and Targacept; and he holds a patent from Repligen. VFG, CCZ, AKT, AD, DJM, EJB and LS declare no conflict of interest.

\section{ACKNOWLEDGEMENTS}

We are thankful to Dr L. Trevor Young and Dr Ana Andreazza to provide target genes for this study. We are also grateful for Natalie Freeman and Maria Tampakeras for technical support.

\section{REFERENCES}

Agostinho FR, Reus GZ, Stringari RB, Ribeiro KF, Ferraro AK, Benedet J et al (2011). Treatment with olanzapine, fluoxetine and olanzapine/fluoxetine alters citrate synthase activity in rat brain. Neurosci Lett 487: 278-281.

Baig MR, Navaira E, Escamilla MA, Raventos H, Walss-Bass C (2010). Clozapine treatment causes oxidation of proteins involved in energy metabolism in lymphoblastoid cells: a possible mechanism for antipsychotic-induced metabolic alterations. J Psychiatr Pract 16: 325-333.

Balijepalli S, Kenchappa RS, Boyd MR, Ravindranath V (2001). Protein thiol oxidation by haloperidol results in inhibition of mitochondrial complex I in brain regions: comparison with atypical antipsychotics. Neurochem Int 38: 425-435.

Barrett JC, Fry B, Maller J, Daly MJ (2005). Haploview: analysis and visualization of LD and haplotype maps. Bioinformatics 21: 263-265.

Ben-Shachar D, Karry R (2008). Neuroanatomical pattern of mitochondrial complex I pathology varies between schizophrenia, bipolar disorder and major depression. PLoS One 3: e3676.

Calle ML, Urrea V, Malats N, Van Steen K (2010). mbmdr: an R package for exploring gene-gene interactions associated with binary or quantitative traits. Bioinformatics 26: 2198-2199.

Choi KH, Higgs BW, Weis S, Song J, Llenos IC, Dulay JR et al (2009). Effects of typical and atypical antipsychotic drugs on gene expression profiles in the liver of schizophrenia subjects. BMC Psychiatry 9: 57.

Da Pozzo E, Costa B, Martini C (2012). Translocator protein (TSPO) and neurosteroids: implications in psychiatric disorders. Curr Mol Med 12: 426-442.

Dudbridge F (2003). Pedigree disequilibrium tests for multilocus haplotypes. Genet Epidemiol 25: 115-121.

Finel M (1998). Organization and evolution of structural elements within complex I. Biochim Biophys Acta 1364: 112-121.

Gaisne M, Bonnefoy N (2006). The COX18 gene, involved in mitochondrial biogenesis, is functionally conserved and tightly regulated in humans and fission yeast. FEMS Yeast Res 6: 869-882.

Gauderman WJ, Morrison JM (2006). A computer program for power and sample size calculations for genetic-epidemiology studies. Available at http://hydra.usc.edu/gxe.

Gebhardt S, Theisen FM, Haberhausen M, Heinzel-Gutenbrunner M, Wehmeier PM, Krieg JC et al (2010). Body weight gain induced by atypical antipsychotics: an extension of the monozygotic twin and sib pair study. J Clin Pharm Ther 35: 207-211.

Gigante AD, Andreazza AC, Lafer B, Yatham LN, Beasley CL, Young LT (2011). Decreased mRNA expression of uncoupling protein 2, a mitochondrial proton transporter, in post-mortem prefrontal cortex from patients with bipolar disorder and schizophrenia. Neurosci Lett 505: 47-51.

Gottesman II, Shields J (1967). A polygenic theory of schizophrenia. Proc Natl Acad Sci USA 58: 199-205.

Graham KA, Perkins DO, Edwards LJ, Barrier RC Jr., Lieberman JA, Harp JB (2005). Effect of olanzapine on body composition and energy expenditure in adults with first-episode psychosis. Am J Psychiatry 162: 118-123.

Hoefs SJ, Skjeldal OH, Rodenburg RJ, Nedregaard B, van Kaauwen EP, Spiekerkotter U et al (2010). Novel mutations in the NDUFS1 gene cause low residual activities in human complex I deficiencies. Mol Genet Metab 100: 251-256. 
Horvath TL, Andrews ZB, Diano S (2009). Fuel utilization by hypothalamic neurons: roles for ROS. Trends Endocrinol Metab 20: 78-87.

Howie B, Fuchsberger C, Stephens M, Marchini J, Abecasis GR (2012). Fast and accurate genotype imputation in genome-wide association studies through pre-phasing. Nature genetics 44: 955-959.

Iuso A, Scacco S, Piccoli C, Bellomo F, Petruzzella V, Trentadue R et al (2006). Dysfunctions of cellular oxidative metabolism in patients with mutations in the NDUFS1 and NDUFS4 genes of complex I. J Biol Chem 281: 10374-10380.

Jordan SD, Konner AC, Bruning JC (2010). Sensing the fuels: glucose and lipid signaling in the CNS controlling energy homeostasis. Cell Mol Life Sci 67: 3255-3273.

Kann O, Kovacs R (2007). Mitochondria and neuronal activity. Am J Physiol Cell Physiol 292: C641-C657.

Konradi C, Eaton M, MacDonald ML, Walsh J, Benes FM, Heckers S (2004). Molecular evidence for mitochondrial dysfunction in bipolar disorder. Arch Gen Psychiatry 61: 300-308.

Lahiri DK, Nurnberger JI Jr. (1991). A rapid non-enzymatic method for the preparation of HMW DNA from blood for RFLP studies. Nucleic Acids Res 19: 5444.

Leloup C, Magnan C, Benani A, Bonnet E, Alquier T, Offer G et al (2006). Mitochondrial reactive oxygen species are required for hypothalamic glucose sensing. Diabetes 55: 2084-2090.

Lett TA, Wallace TJ, Chowdhury NI, Tiwari AK, Kennedy JL, Muller DJ (2012). Pharmacogenetics of antipsychotic-induced weight gain: review and clinical implications. Mol Psychiatry 17: 242-266.

Lieberman JA, Stroup TS, McEvoy JP, Swartz MS, Rosenheck RA, Perkins DO et al (2005). Effectiveness of antipsychotic drugs in patients with chronic schizophrenia. New Engl J Med 353: 1209-1223.

Maurer I, Zierz S, Moller H (2001). Evidence for a mitochondrial oxidative phosphorylation defect in brains from patients with schizophrenia. Schizophr Res 48: 125-136.

Muller DJ, Kennedy JL (2006). Genetics of antipsychotic treatment emergent weight gain in schizophrenia. Pharmacogenomics 7: 863-887.

Nyholt DR (2004). A simple correction for multiple testing for single-nucleotide polymorphisms in linkage disequilibrium with each other. Am J Hum Genet 74: 765-769.

Prabakaran S, Swatton JE, Ryan MM, Huffaker SJ, Huang JT, Griffin JL et al (2004). Mitochondrial dysfunction in schizo- phrenia: evidence for compromised brain metabolism and oxidative stress. Mol Psychiatry 9: 684-697 643.

Purcell S, Neale B, Todd-Brown K, Thomas L, Ferreira MA, Bender D et al (2007). PLINK: a tool set for whole-genome association and population-based linkage analyses. Am J Hum Genet 81: 559-575.

Reinke A, Martins MR, Lima MS, Moreira JC, Dal-Pizzol F, Quevedo J (2004). Haloperidol and clozapine, but not olanzapine, induces oxidative stress in rat brain. Neurosci Lett 372: $157-160$.

Scola G, Kim HK, Young LT, Andreazza AC (2013). A fresh look at complex I in microarray data: clues to understanding diseasespecific mitochondrial alterations in bipolar disorder. Biol Psychiatry 73: e4-e5.

Smeitink JA, Loeffen JL, Triepels RH, Smeets RJ, Trijbels JM, van den Heuvel LP (1998). Nuclear genes of human complex I of the mitochondrial electron transport chain: state of the art. Hum Mol Genet 7: 1573-1579.

Sun L, Rommens JM, Corvol H, Li W, Li X, Chiang TA et al (2012). Multiple apical plasma membrane constituents are associated with susceptibility to meconium ileus in individuals with cystic fibrosis. Nat Genet 44: 562-569.

Sun X, Wang JF, Tseng M, Young LT (2006). Downregulation in components of the mitochondrial electron transport chain in the postmortem frontal cortex of subjects with bipolar disorder. J Psychiatry Neurosci 31: 189-196.

Team RC (2012). R: A Language and Environment for Statistical Computing. $R$ Foundation for Statistical Computing.

Tiwari AK, Brandl EJ, Weber C, Likhodi O, Zai CC, Hahn MK et al (2013). Association of a functional polymorphism in neuropeptide y with antipsychotic-induced weight gain in schizophrenia patients. J Clin Psychopharmacol 33: 11-17.

Walss-Bass C, Weintraub ST, Hatch J, Mintz J, Chaudhuri AR (2008). Clozapine causes oxidation of proteins involved in energy metabolism: a possible mechanism for antipsychotic-induced metabolic alterations. Int J Neuropsychopharmacol 11: 1097-1104.

Ward LD, Kellis M (2012). HaploReg: a resource for exploring chromatin states, conservation, and regulatory motif alterations within sets of genetically linked variants. Nucleic Acids Res 40(Database issue): D930-D934.

Xu Z, Taylor JA (2009). SNPinfo: integrating GWAS and candidate gene information into functional SNP selection for genetic association studies. Nucleic Acids Res 37(Web Server issue): W600-W605.

Supplementary Information accompanies the paper on the Neuropsychopharmacology website (http://www.nature.com/npp) 